Supporting Information:

\title{
Design of a microfluidic chip for magnetic-activated sorting of one-bead- one-compound libraries
}

Choi-Fong Cho ${ }^{1}$, Kyungheon Lee ${ }^{2}$, Maria-Carmela Speranza ${ }^{1}$, Fernanda C. Bononi ${ }^{3}$, Mariano S.

Viapiano ${ }^{1}$, Leonard G. Luyt ${ }^{3}$, Ralph Weissleder ${ }^{2}$, E. Antonio Chiocca ${ }^{1}$, Hakho Lee ${ }^{2}$, Sean E. Lawler ${ }^{1}$.

1. Harvey Cushing Neuro-Oncology Laboratories, Department of Neurosurgery, Brigham and Women's Hospital, Harvard Medical School, Boston, MA 02115 (USA).

2. Center for Systems Biology, Massachusetts General Hospital, Harvard Medical School, Boston, MA 02114 (USA)

3. Departments of Chemistry and Oncology, University of Western Ontario, London, ON N6A 3K7 (Canada)

Correspondence: Choi-Fong Cho, Ph.D.

Department of Neurosurgery, Brigham and Women's Hospital, Harvard Medical School.

4 Blackfan Circle, HIM 9-07

Boston, MA 02115, U.S.

Email: ccho@bwh.harvard.edu 

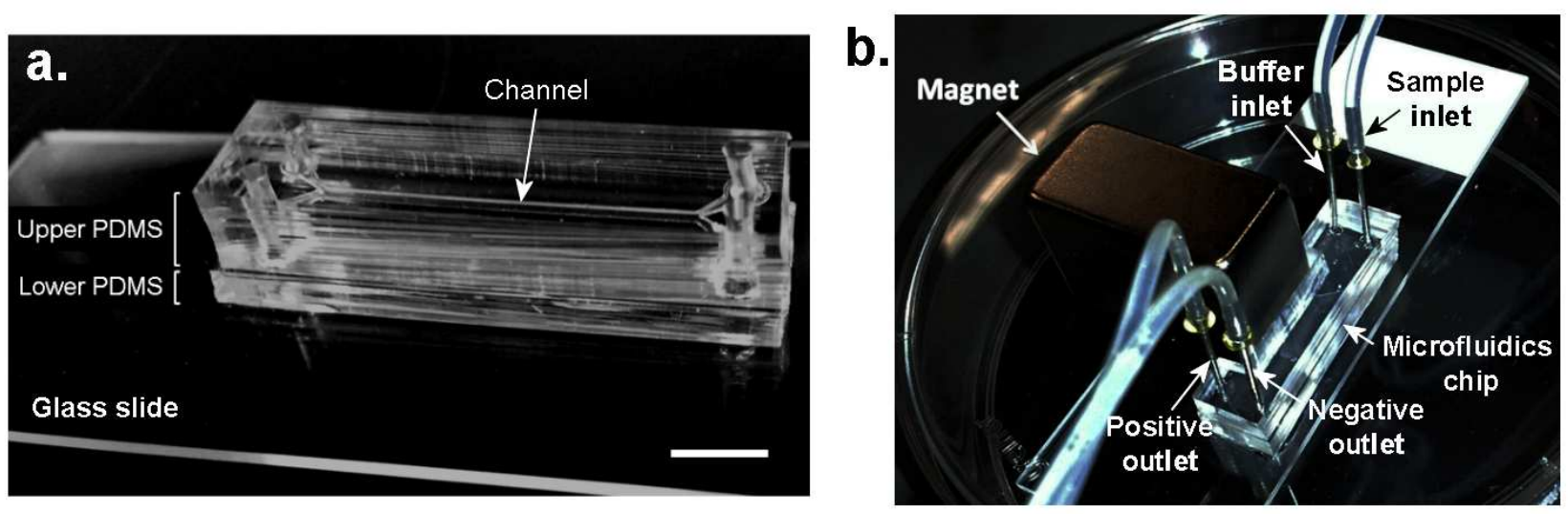

Supplementary Figure 1. Construction of microfluidic magnetic sorter. (a) Two identical openfaced PDMS channels (upper and lower PDMS) were aligned precisely and bonded to form the microfluidic device. Scale bar, $5 \mathrm{~mm}$. (b) An external neodymium magnet was placed adjacent to the collection flow path. (Optional: Excess PDMS adjacent to the collection flow path can be excised using a sharp razor blade to allow placement of the magnet in closer proximity with the channel). 
Supplementary Figure 2. Magnetic sorting using microfluidic device. Time-lapse video microscopy of the microfluidics channel and its outlets showing the sorting of a mixed population of positive (magnetized) and control (non-magnetized) beads. (Red: Magnetic particles; White: Control beads).

(Contains web-enhanced object content) 